\title{
Protocol Summary for Pharmacogenomic Assessments Domain
}

National Cancer Institute

\section{Source}

National Cancer Institute. Protocol Summary for Pharmacogenomic Assessments

Domain. NCl Thesaurus. Code C111300.

A special purpose domain that contains the protocol summary information for pharmacogenomics assessments. 